\title{
Moduli space metric for maximally-charged dilaton black holes
}

\author{
Kiyoshi Shiraishi \\ Akita Junior College, Shimokitade-Sakura, Akita-shi, Akita 010, Japan \\ Nuclear Physics B402 (1993) pp. 399-410
}

\begin{abstract}
The system consisting of slowly-moving, maximally charged, nonrotating dilaton black holes is investigated. We obtain the metric on the moduli space of the system in the low-velocity limit. We find that: (1) only two-body interactions exist between the extreme black holes in string theory; (2) the mutual interaction between the black holes vanishes if the dilaton can be interpreted as a Kaluza-Klein scalar; and (3) for general dilaton couplings, there exist many-body interactions among the extreme black holes. We analyze the low-energy classical scattering of the two extreme black holes in string theory by utilizing the moduli space metric.
\end{abstract}

\section{Introduction}

Recently, it is very fashionable to study the properties of exact soliton solutions in gravitating systems where dilaton couplings exist. A context of the investigation originates from the study of string theory [1]. In this case, the dilaton coupling and the other interactions in the effective field theory are readily determined by the string theory. On the other hand, there have been many works on the study of charged black holes in the theory with arbitrary dilaton coupling $[2,3,4,5,6,7]$. Through the study on the properties of black holes, we have learned the fact that the value of dilaton coupling in string theory (and possibly in the case with no dilaton coupling $[3,4]$ ) is a critical value for thermodynamics of black holes.

The static multi-black-hole solution in the dilaton-coupled Einstein-Maxwell system has been obtained by the present author in ref. [7]. This solution is a generalization of the Papapetrou-Majumdar-Myers solution [8]. We found the balance of static forces in the multi-black-hole system described by the solution. The forces are the Coulomb repulsive force, gravitational force, and attractive scalar force. In ref. [7], we have studied the interaction between the slowlymoving extremal holes using the Lienard-Wiechert method at large distances $[9,10,11]$. We have found that a special value of the dilaton coupling reduces the total force to nothing. 
In the present paper, we study the motion of the maximally charged dilaton black holes in the low-velocity limit by calculating an effective action for the system. The method to obtain the action was originally developed by Ferrell and Eardley [12]. We manage to extend their method to the dilaton-coupled system. The effective action reveals the many-body interaction between the slowly moving extremal black holes at arbitrary distances. The strength as well as the nature of the interaction may depend on the value of the dilaton coupling. One may find the peculiar feature appearing in string theory.

The organization of this paper is as follows.

We show the static multi-centered solution in the $(1+N)$-dimensional dilatoncoupled system in the subsequent section. Though the exact solution has already been shown in ref. [7], we recapitulate it here because it is crucial for the calculation in the following sections.

In sect. 3, we derive the effective action for the multi-black-hole system of the slow motion. We obtain the exact metric on the moduli space and discuss the low-energy scattering of two extreme black holes in string theory in sect. 4 .

The last section is devoted to conclusions.

\section{The multi-dilaton-black-hole solution}

We begin with writing the action for classical fields which we study here. It takes the following form (without source terms):

$$
S=\int d^{N+1} x \frac{\sqrt{-g}}{16 \pi}\left(R-\frac{4}{N-1}(\nabla \phi)^{2}-e^{-[4 a /(N-1)] \phi} F^{2}\right) \quad(N \geq 3),
$$

where we fix the Newton constant to unity. The coupling constant $a$ (which can be taken as non-negative) is the parameter which determines the strength of the coupling between the Maxwell field $F$ and the dilaton field $\phi$.

For $a=0$, the action is reduced to the one for the usual Einstein-Maxwell system with a free scalar field. In this case, multi-centered static solutions to the Einstein-Maxwell equations were found by Papapetrou and Majumdar and by Myers [8]. Their solutions describe many-body systems of extremely charged black holes, where the gravitational attraction and Coulomb repulsion is exactly cancelled in the static configuration.

For $a=1$, the action reduces to the one which is derived from the lowenergy string theory $[1,2,3]$. For $a=1$, one can use the "stringy" metric $\bar{g}_{\mu \nu}=\exp [4 \phi /(N-1)] g_{\mu \nu}$ to get the action in a familiar form,

$$
S=\int d^{N+1} x \frac{\sqrt{-g}}{16 \pi} e^{-2 \phi}\left[\bar{R}+4(\bar{\nabla} \phi)^{2}-F^{2}\right] .
$$

The cases with general dilaton couplings are examined in ref. [7]. The metric for the $n$-black-hole solution is found to be

$$
d s^{2}=-U^{-2}(\mathbf{x}) d t^{2}+U^{2 /(N-2)}(\mathbf{x}) d \mathbf{x}^{2},
$$


where

$$
U(\mathbf{x})=(F(\mathbf{x}))^{(N-2) /\left(N-2+a^{2}\right)},
$$

and

$$
F(\mathbf{x})=1+\sum_{i=1}^{n} \frac{\mu_{i}}{(N-2)\left|\mathbf{x}-\mathbf{x}_{i}\right|^{N-2}} .
$$

The (Maxwell) vector one-form and the dilaton configuration are written as

$$
A= \pm \sqrt{\frac{N-1}{2\left(N-2+a^{2}\right)}}(F(\mathbf{x}))^{-1} d t,
$$

and

$$
e^{-[4 a /(N-1)] \phi}=(F(\mathbf{x}))^{2 a^{2} /\left(N-2+a^{2}\right)} .
$$

In this solution, the asymptotic value of $\phi$ is fixed to be zero.

If we set $a=0$ in the solution, we find that the solution reduces to the Papapetrou-Majumdar-Myers solution in $1+N$ dimensions [8].

The mass and electric charge corresponding to each point source $(=$ extreme black hole) is $[2,7]$

$$
\begin{aligned}
m_{i} & =\frac{A_{N-1}(N-1)}{8 \pi\left(N-2+a^{2}\right)} \mu_{i}, \\
\left|Q_{i}\right| & =\sqrt{\frac{N-1}{2\left(N-2+a^{2}\right)}} \mu_{i},
\end{aligned}
$$

where $A_{N-1}=2 \pi^{N / 2} / \Gamma(N / 2)$.

We can define the scalar charge $\sigma$ by examining the asymptotic behavior of the dilaton $[2,7]$. The scalar charge associated with each source is

$$
\left|\sigma_{i}\right|=\sqrt{\frac{1}{2}(N-1)} \frac{a}{N-2+a^{2}} \mu_{i} .
$$

Now we can examine the balance in the static forces. The system under consideration is governed by three kinds of forces; the newtonian attraction, the Coulomb repulsion, and the attractive scalar force mediated by the dilaton. One can easily confirm the fact that the total force vanishes in the system described by the static solution [7].

We anticipate that an addition of a small amount of kinetic energy to this static system can be treated by perturbation. In such a case, radiation reactions can be ignored, because the intensity of the radiations are generally proportional to the higher order in the velocities. The Lienard-Wiechert potential method has been applied to the system in ref. [7]. We exhibit the two-body result in appendix $\mathrm{A}$ in the present paper. By using this method, we know the properties and strength of the interactions between sources with arbitrary amounts of charges, but we can hardly get the information of the interaction at small distances.

In the next section, we apply the method of Ferrell and Eardley [12] to the system of extreme charged dilaton black holes to get the information on the slow motion of the holes at arbitrary distances. 


\section{The effective action for the many-body system of maximally-charged dilaton black holes in the low-velocity limit}

In this section we follow the procedure of ref. [12], based on the static solution (2). We must also be careful not to miss the contribution from the dilaton field. First we calculate the classical fields in the presence of the slowly-moving blackhole sources perturbatively. Second, substituting the perturbative solutions into the action for the classical field, we obtain the effective action for maximallycharged dilaton black holes.

First, let us consider the off-diagonal part of the metric and the space components of the gauge field simultaneously. We need only the $O(v)$ perturbative solution for these fields to obtain the effective Lagrangian up to $O\left(v^{2}\right)[12]$ (Here $v$ represents the velocity of the black hole as a point source.) The perturbed metric and potential are written in the form

$$
\begin{gathered}
d s^{2}=-U^{-2}(\mathbf{x}) d t^{2}+2 \mathbf{N} \cdot d \mathbf{x} d t+U^{2 /(N-2)}(\mathbf{x}) d \mathbf{x}^{2} \\
A=\sqrt{\frac{N-1}{2\left(N-2+a^{2}\right)}}(F(\mathbf{x}))^{-1} d t+\mathbf{A} \cdot d \mathbf{x}
\end{gathered}
$$

where $U$ and $F(\mathbf{x})$ are defined by (3) and (4). We have only to solve linearized equations with perturbed sources up to $O(v)$ for $N_{i}$ and $A_{i}$. We should note that in the system, the action for changed point sources (particles) coupled to the dilaton field is formally written as

$$
\sum_{i=1}^{n} \int d s_{i}\left(m_{i} e^{[2 a /(N-1)] \phi}+Q_{i} A_{\mu} \frac{\partial x_{i}^{\mu}}{\partial s_{i}}\right),
$$

which is also checked by looking back on the solution (2). The coupling between the vector field and the source does not suffer from the effect of dilaton coupling.

For notational simplicity as well as gauge invariance, we use the one-forms $P$ and $Q$ which consist of the combination of $N$ and $A$ :

$$
\begin{gathered}
P=A+\sqrt{\frac{N-1}{2\left(N-2+a^{2}\right)}}(F(\mathbf{x}))^{-1} Q, \\
Q=U^{2} N .
\end{gathered}
$$

Keeping the first-order terms in $P$ and $Q$, we obtain the similar equations to those in ref. [12]. Consequently the solutions are found to be

$$
\begin{gathered}
F^{2\left(a^{2}-1\right) /\left(N-2+a^{2}\right)} d P=-\frac{N-a^{2}}{N-2+a^{2}} \sum_{i=1}^{n} Q_{i} d f_{i} \wedge \mathbf{v}_{i} \cdot d \mathbf{x}_{i} \\
F^{\left(a^{2}-N\right) /\left(N-2+a^{2}\right)} d Q=-4 \sqrt{\frac{N-1}{2\left(N-2+a^{2}\right)}} \sum_{i=1}^{n} Q_{i} d f_{i} \wedge \mathbf{v}_{i} \cdot d \mathbf{x}_{i}
\end{gathered}
$$


where $d f$ and $\mathbf{v} \cdot d \mathbf{x}$ are one-forms and

$$
f_{i}=\frac{1}{(N-2)\left|\mathbf{x}-\mathbf{x}_{i}\right|^{N-2}} .
$$

We substitute (15) and (16) into the field-theory action with the boundary term for the Einstein gravity. The second-derivatives of the fields are eliminated by adding the boundary term. In addition, we must also pay attention to the perturbation on the dilaton kinetic term and source terms.

Synthesizing all the contributions of low-velocity perturbation, we obtain the effective Lagrangian up to $O\left(v^{2}\right)$ for $n$ maximally-charged dilaton black holes,

$$
\begin{aligned}
L= & -\sum_{i=1}^{n} m_{i}+\sum_{i=1}^{n} m_{i}\left(v_{i}\right)^{2}+\frac{(N-1)\left(N-a^{2}\right)}{16 \pi\left(N-2+a^{2}\right)^{2}} \\
& \times \int d^{N} \mathbf{x}(F(\mathbf{x}))^{2\left(1-a^{2}\right) /\left(N-2+a^{2}\right)} \sum_{i, j}^{n} \frac{\left(\mathbf{n}_{j} \cdot \mathbf{n}_{j}\right)\left|\mathbf{v}_{i}-\mathbf{v}_{j}\right|^{2} \mu_{i} \mu_{j}}{2\left|\mathbf{r}_{i}\right|^{N-1}\left|\mathbf{r}_{j}\right|^{N-1}}
\end{aligned}
$$

where $\mathbf{r}_{i}=\mathbf{x}-\mathbf{x}_{i}$ and $\mathbf{n}=\mathbf{r}_{i} /\left|\mathbf{r}_{i}\right| . F(\mathbf{x})$ is defined by (4).

Here the background dilaton contribution in the free part of the action is already regularized. The integration must be carried out with care for the regularization of divergences and seemingly-divergent integrals.

If we send the dilaton coupling to zero and set $N=3$ in the above expression, we reproduce the result of ref. [12]. (The last term in eq. (10b) in ref. [12] has no effect in the integration.) Furthermore, in the large-separation limit (where we approximate $F(x)$ by one), we find exactly the same result as gained by the Lienard-Wiechert method [7] (see appendix A).

It can directly be observed that the interaction part of the Lagrangian vanishes when $a^{2}=N$. This value of the coupling corresponds to Kaluza-Klein reduction of $(N+2)$-dimensional space-time to $(N+1)$-dimensional space-time with a circle $\left(S^{1}\right)[2]$.

A more interesting point we soon become aware of is the existence of manybody interactions. In general, we obtain infinite species of many-body interactions by expanding the function $\mathrm{F}(\mathrm{x})$. Some special cases arise: (1) when $a^{2}=0$ and $N=3$, the black holes are governed only by two-body, three-body, and four-body interactions [12], (2) when $a^{2}=0$ and $N=4$, there are only two-body and three-body interactions, (3) when $a^{2}=1$ (and any $N$ ), there are only two-body interactions, and (4) when $a^{2}=N$, there are no interactions.

Therefore in low-energy string theory, there exist only two-body interactions between maximally-charged black holes, regardless of the dimension of spacetime. This result seems most interesting, and suggests the necessity of further study of the exact solutions and string theory [1].

In the next section, we will study the moduli space and the scattering of two slowly-moving black holes laying particular emphasis on the string case $\left(a^{2}=1\right)$. 


\section{The moduli space metric and the slow motion of two maximally charged black holes in string theory}

We wish to study the slow motion of the maximally-charged dilaton black holes in the low-energy limit. Here the low-energy limit means the situation where any radiation reaction can be ignored.

The slow motion of classical lumps or solitons in many kinds of field theoretical models is expressed by geodesic motion on the moduli space, which is the space of the parameters in the static configuration $[13,14,15]$. For the system consisting of multi-centered extreme Reissner-Nordstrom black holes, the metric of the moduli space was studied in refs. [10, 12].

In the presence of general scalar interactions, the moduli space metric can be obtained from the expression (3.9). In this section, we concentrate our attention to the string case, $a^{2}=1$.

For $a^{2}=1$, the interaction part in the Lagrangian becomes very simple and after integration we get

$$
L=-\sum_{i} m_{i}+\sum_{i} \frac{1}{2} m_{i} v_{i}^{2}+\frac{A_{N-1}}{16 \pi(N-2)} \sum_{i, j} \frac{\left|\mathbf{v}_{i}-\mathbf{v}_{j}\right|^{2} \mu_{i} \mu_{j}}{2 r_{i j}^{N-2}}
$$

where $r_{i j}=\left|\mathbf{x}_{i}-\mathbf{x}_{j}\right|$ and

$$
m_{i}=\frac{A_{N-1}}{8 \pi} \mu_{i} .
$$

Now we focus our attention on the two-black-hole system. The Lagrangian becomes

$$
L_{2 B}=-M+\frac{1}{2} M V^{2}+\frac{1}{2} \mu v^{2}\left(1+\frac{8 \pi M}{A_{N-1}(N-2) r^{N-2}}\right),
$$

where $M=m_{a}+m_{b}, \mu=m_{a} m_{b} / M, \mathbf{V}=\left(m_{a} \mathbf{v}_{a}+m_{b} \mathbf{v}_{b}\right) / M, \mathbf{v}=\mathbf{v}_{a}-\mathbf{v}_{b}$ and $r=\left|\mathbf{x}_{a}-\mathbf{x}_{b}\right|$.

Thus the metric of the $2 N$-dimensional moduli space for the two-body system turns out to be

$$
d s^{2}=M d \mathbf{R}^{2}+\mu\left(1+\frac{8 \pi M}{A_{N-1}(N-2) r^{N-2}}\right) d \mathbf{r}^{2},
$$

where $\mathbf{R}=\left(m_{a} \mathbf{x}_{a}+m_{b} \mathbf{x}_{b}\right) / M$ and $\mathbf{r}=\mathbf{x}_{a}-\mathbf{x}_{b}$.

The center of mass moves freely, as usually expected. Moreover, we consider a two-dimensional intersection of the moduli space for simplicity. The two parameters are the distance between two black holes $(r)$ and the azimuthal angle $(\theta)$ on the scattering plane.

The global structure of this surface of the moduli space depends on the spatial dimension $N$. The reduced metric can be written in the form

$$
d s_{M S}^{2}=g(r)\left(d r^{2}+r^{2} d \theta^{2}\right)=h(R) d R^{2}+R^{2} d \theta^{2},
$$


where

$$
g(r)=1+\frac{8 \pi M}{A_{N-1}(N-2) r^{N-2}},
$$

and $R(r)$ is a new radial coordinate. $\mu$ has been absorbed in the rescaling of $r$.

We can realize the surface immersed in the three-dimensional euclidean space spanned by the coordinates $R, \theta$, and $z(R)$. Such a surface for the case with $N=3$ and $a=0$ was exhibited in ref. [12]. The "throat" of the surface is located at the distance where $h(R(r))$ diverges.

In the present $a^{2}=1$ case, for $N>4$, the throat is located at

$$
r=\left(\frac{4 \pi(N-4) M}{A_{N-1}(N-2)}\right)^{1 /(N-2)} .
$$

The surface looks like fig. 1 of ref. [12] in this case (fig. 1a).

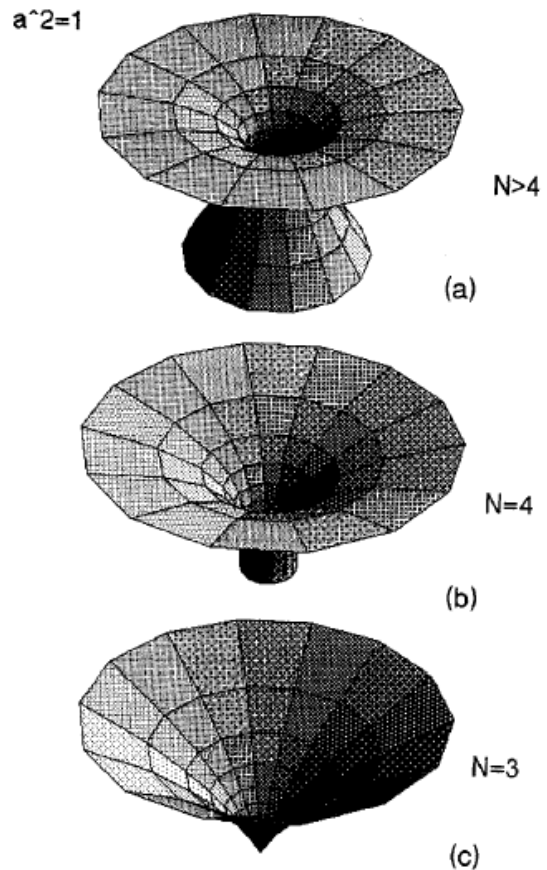

Figure 1: A schematic view of the geometry of the moduli space of the two-body system of extreme black holes in string theory $\left(a^{2}=1\right)$. (a) for the case $N>4$, (b) for the case $N=4$, and (c) for the case $N=3$.

In the $a^{2}=1$ case, the surface of the moduli does not have a throat if $N=3$ or 4 . For $N=4$, the surface near the origin looks like a "tube", and the origin $r=0$ corresponds to the infinity far down the tube (fig. 1b). 
For $N=3$, the surface has the shape such as fig. 1c. There is a deficit angle $r$ around the origin of the moduli space for the two-body problem, while the moduli space in the asymptotic region approaches a flat space with no deficit angle. Suppose that the two black holes approach each other from spatial infinity with low relative velocity and impact parameter $b$. For $N>4$ (and $\left.a^{2}=1\right)$, the two black holes coalesce if $b$ is smaller than the critical value

$$
b_{\text {coal }}=\sqrt{\frac{N-2}{N-4}}\left(\frac{4 \pi(N-4) M}{A_{N-1}(N-2)}\right)^{1 /(N-2)} \quad(N>4),
$$

which is in the same order of the radius of the throat.

For $N=4$ and $a^{2}=1$, the critical value for $b$ is given by

$$
b_{\text {coal }}=\sqrt{\frac{2 M}{\pi}} \quad(N=4) .
$$

For $N=3$ and $a^{2}=1$, the black holes never coalesce according to the geodesic approximation on the moduli space.

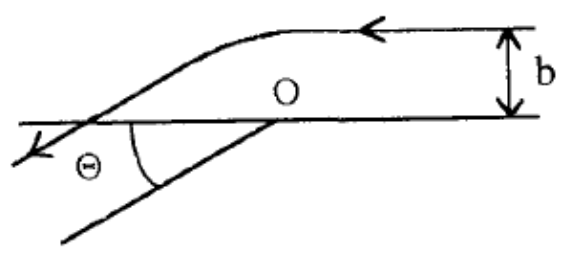

Figure 2: The deflection angle $\Theta$.

For $N=3$ and $N=4$, the deflection angle (see fig. 2) can be exactly calculated. The results are the following $\left(b>b_{\text {coal }}\right.$ for $\left.N=4\right)$

$$
\begin{aligned}
& \Theta=2 \tan ^{-1} \frac{M}{b} \quad\left(N=3 \text { and } a^{2}=1\right), \\
& \Theta=\pi\left[\left(1-\frac{2 M}{\pi b^{2}}\right)^{-1 / 2}-1\right]\left(b>b_{\text {coal }}, N=4 \text { and } a^{2}=1\right) .
\end{aligned}
$$

In the case of $N=3$, the naive limit $b \rightarrow 0$ yields $\Theta=\pi$, i.e., the backward scattering. This behavior can be derived from the nature of the metric of the moduli space. (The scattering of two extreme black holes in four-dimensional string theory appears much alike that of two BPS monopoles [13, 15]; a crucial difference is, however, the fact that we can tell the distinction of two black holes.) By now, however, we do not know the applicability of the low-energy regime to the case with small impact parameter.

The differential cross section in this $N=3$ case turns out to be in the form of the Rutherford scattering,

$$
\frac{d \sigma}{d \Omega}=\frac{1}{4} \frac{M^{2}}{\sin ^{4}\left(\frac{1}{2} \Theta\right)} .
$$


The analysis of higher-dimensional cases and of the case with general dilaton coupling, which is of much importance, can onlybe done by the help of numerical calculations. This is beyond the scope of the present paper.

\section{Conclusion}

We have obtained the effective action for the system consisting of $n$ maximally charged dilaton black holes in the low-velocity limit. The analysis has been performed in $1+N$ dimensions $(N>3)$ and is valid at small distances.

We have found a peculiar feature appearing in string theory. Further, the $N=3$ case is a special case in that the two extreme black holes never merge in the low-velocity regime. We should also notice that the slow motion of the black holes depends only on the total mass in the two-body case.

Finally we guess that the exact metric for the moduli space can be obtained by some other way(s) in the case with particular values of dilaton couplings, such as $a^{2}=N$ and $a^{2}=1$, beyond the low-energy regime. We wish to study this conjecture in the future.

\section{Appendix A}

Here we show the low-energy effective Lagrangian $[9,10,11]$ for the system which consists of two point sources separated at a large distance with general amounts of masses, electric charges, and scalar charges. (The earlier version of the preprint of ref. [7] contains errors in some coefficients.) The Lagrangian which contains up to $O\left(v^{2}\right)$ terms is written in the form

$$
\begin{aligned}
L= & \frac{1}{2} m_{a} v_{a}+\frac{1}{2} m_{b} v_{b}+\frac{A_{N-1}}{4 \pi(N-2) R_{a b}^{N-2}} \\
& \times\left[Q_{a} Q_{b}-\sigma_{a} \sigma_{b}-\left(\frac{4 \pi}{A_{N-1}}\right)^{2} \frac{2(N-2)}{N-1} m_{a} m_{b}\right]+\frac{A_{N-1}\left(v_{a}^{2}+v_{b}^{2}\right)}{8 \pi(N-2) R_{a b}^{N-2}} \\
& \times\left[-\sigma_{a} \sigma_{b}+\left(\frac{4 \pi}{A_{N-1}}\right)^{2} \frac{2 N}{N-1} m_{a} m_{b}\right]+\frac{A_{N-1}\left(\mathbf{v}_{a} \cdot \mathbf{v}_{b}\right)}{8 \pi(N-2) R_{a b}^{N-2}}\left[Q_{a} Q_{b}+\sigma_{a} \sigma_{b}\right. \\
& \left.-\left(\frac{4 \pi}{A_{N-1}}\right)^{2} \frac{2(3 N-2)}{N-1} m_{a} m_{b}\right]+\frac{A_{N-1}\left(\mathbf{n} \cdot \mathbf{v}_{b}\right)\left(\mathbf{n} \cdot \mathbf{v}_{b}\right)}{8 \pi(N-2) R_{a b}^{N-2}} \\
& \times\left[(N-2) Q_{a} Q_{b}-(N-2) \sigma_{a} \sigma_{b}-\left(\frac{4 \pi}{A_{N-1}}\right)^{2} \frac{2(N-2)^{2}}{N-1} m_{a} m_{b}\right]+\cdot(31)
\end{aligned}
$$

where $R_{a b}$ is the distance between two point sources denoted $a$ and $b$, and $\mathbf{n}$ is the unit vector in the direction $b-a$.

When the relation of the extreme charged dilaton black hole (7), (8), (9) are 
substituted, we find

$$
L=\frac{1}{2} m_{a} v_{a}^{2}+\frac{1}{2} m_{b} v_{b}^{2}+\frac{A_{N-1}(N-1)\left(N-a^{2}\right)\left|\mathbf{v}_{a}-\mathbf{v}_{b}\right|^{2} \mu_{a} \mu_{b}}{16 \pi(N-2)\left(N-2+a^{2}\right)^{2} R_{a b}^{N-2}}+\ldots,
$$

which precisely recovers the result obtained in the text in the large-distance limit ( $F$ is replaced by one).

\section{References}

[1] A. Dabholkar et al., Nucl. Phys. B340 (1990) 33;

A. Strominger, Nucl. Phys. B343 (1990) 167;

E. Copeland, D. Haws and M. Hindmarsh, Phys. Rev. D42 (1990) 726;

M. J. Duff and J. X. Lu, Phys. Rev. Lett. 66 (1991) 1402; Nucl. Phys. B354 (1991) 129, 141; B357 (1991) 534; Phys. Lett. B273 (1991) 409;

M. J. Duff and K. S. Stelle, Phys. Lett. B253 (1991) 113;

R. Guven, Phys. Lett. B276 (1992) 49;

R. R. Khuri, Phys. Lett. B259 (1991) 261;

C. G. Callan, Jr. and R. R. Khuri, Phys. Lett. B261 (1991) 363;

C. G. Callan Jr., J. A. Harvey and A. Strominger, Nucl. Phys. B359 (1991) 611; B367 (1991) 60;

G. T. Horowitz and A. Strominger, Nucl. Phys. B360 (1991) 197;

J. A. Harvey and A. Strominger, Phys. Rev. Lett. 66 (1991) 549;

S. B. Giddings and A. Strominger, Phys. Rev. Lett. 67 (1991) 2930;

D. Brill and G. T. Horowitz, Phys. Lett. B262 (1991) 437;

I. Martin and A. Restuccia, Phys. Lett. B271 (1991) 361;

J. Home, G. Horowitz and A. Steif, Phys. Rev. Lett. 68 (1992) 568;

J. H. Horne and G.T. Horowitz, Nucl. Phys. B368 (1992) 444;

J.A. Harvey and J. Liu, Phys. Lett B268 (1991) 40.

[2] G.W. Gibbons and K. Maeda, Nucl. Phys. B298 (1988) 741.

[3] D. Garfinkle, G. Horowitz and A. Strominger, Phys. Rev. D43 (1991) 3140; D45 (1992) 3888 (E).

[4] J. Preskill, P. Schwarz, A. Shapere, S. Trivedi and F. Wilczek, Mod. Phys. Lett. A6 (1991) 2353.

[5] C. F. E. Holzhey and F. Wilczek, Nucl. Phys. B380 (1992) 447.

[6] K. Shiraishi, Phys. Lett. A166 (1992) 298.

[7] K. Shiraishi, J. Math. Phys. 34 (1993) 1480 (arXiv:1402.5484). 
[8] A. Papapetrou, Proc. R. Irish Acad. A51 (1947) 191;

S. D. Majumdar, Phys. Rev. 72 (1947) 930;

R. C. Myers, Phys. Rev. D35 (1987) 455.

[9] P. J. Ruback, Commun. Math. Phys. 107 (1986) 93.

[10] G. W. Gibbons and P. J. Ruback, Phys. Rev. Lett. 57 (1986) 1492.

[11] L. D. Landau and E. M. Lifschitz, Classical theory of fields, 4th ed. (Pergamon, Oxford, 1975).

[12] R. C. Ferrell and D. M. Eardley, Phys. Rev. Lett. 59 (1987) 1617.

[13] N. S. Manton, Phys. Lett. B110 (1982) 54.

[14] R. Ward, Phys. Lett. B158 (1985) 424.

[15] M. Atiyah and N.J. Hitchin, The geometry and dynamics of magnetic monopoles (Princeton U. P., Princeton, NJ, 1988). 